\title{
Preface: Special functions and codes
}

\author{
Cunsheng Ding ${ }^{1} \cdot$ Zhengchun Zhou $^{2}$
}

Published online: 22 August 2016

(C) Springer Science+Business Media New York 2016

In the past two decades, we have witnessed a lot of interplays between special functions and codes. In one direction, special codes were employed to construct functions with desirable properties. A very successful example in this direction is the Carlet-Feng construction of an infinite family of balanced Boolean functions having good cryptographic properties with the Reed-Solomon codes (see, Proceedings of ASIACRYPT 2008, LNCS 5350, pp. 425-440, 2008, Springer Verlag). The BCH bound plays an important role in proving the degree of the algebraic immunity of the Carlet-Feng family of Boolean functions. In the other direction, many special functions were used in different ways for constructing optimal linear codes. For instance, almost perfect nonlinear functions, planar functions and Dickson polynomials were employed to produce optimal linear codes in many references. These nice and impressive interplays between special functions and codes are the driving force of this special issue, which includes ten rigorously selected papers from the original submissions.

The weight distribution of linear codes has been a hot research topic in coding theory. It not only shows the error-correcting capability of a linear code, but also gives much more information about the code. It has applications in association schemes, authentication codes, combinatorial designs, and secret sharing, to mention only a few. It is in general very difficult to determine the minimum distance of a linear code, let alone its weight distribution. The determination of the complete weight distribution of nonbinary linear codes is harder. However, in certain special cases, the weight distribution of a linear code may be settled. For instance, this is possible when the dimension of a code is very small compared with

Cunsheng Ding

cding @ust.hk

Zhengchun Zhou

zzc@home.swjtu.edu.cn

1 Department of Computer Science and Engineering,

The Hong Kong University of Science and Technology,

Clear Water Bay, Kowloon, Hong Kong, China

2 School of Mathematics, Southwest Jiaotong University,

Chengdu, 610031, China 
its length. In the past two decades, a lot of progress on the weight distribution of linear codes has been made. Six papers in this special issue deal with the weight distribution or the complete weight distribution of some families of linear codes over finite fields, which are defined by special functions.

Linear codes with only a few weights are interesting in theory for many reasons. For example, projective two-weight codes and strongly regular graphs are equivalent, and binary three-weight codes have been employed to construct association schemes. In applications, they are also very attractive. For instance, linear codes with a few weights have applications in authentication codes and secret sharing. Most recently, some three-weight and fiveweight codes have been used to construct exponentially many infinite families of 2-designs and 3-designs. Six papers in this special issue present linear codes with a few weights. The linear codes in the six papers are based on a fundamental construction, where the trace function plays a key role. In addition, these codes are deeply related to quadratic functions, planar functions, monomials and binomials. This is why the weight distributions of these codes have been settled in these papers.

Bent functions have been used to construct codes in a number of ways in the literature. Four of the papers in this special issue involve bent functions. One of them (i.e., the paper by $\mathrm{Z}$. Heng and $\mathrm{Q}$. Yue) employs bent functions over the ring $\mathrm{Z}_{4}$ to construct codebooks meeting the Levenshtein bound.

Goppa codes are named after Valerii Denisovich Goppa. They form a large class of linear codes, and include the narrow-sense BCH codes as a subclass. Though Goppa codes have a long history, little is known about their dimension and minimum distance. Similar to BCH codes, there are lower bounds on both the dimension and the minimum distance of Goppa codes. But these bounds are not tight in general. However, if the Goppa polynomial has speciality and the location set is properly chosen, the classical bounds on the dimension and the minimum distance may be improved for the corresponding Goppa code. The paper by S. Bezzateev and N. Shekhunova goes in this direction.

The paper by Q. Wang determines the dimension and generator polynomial of some cyclotomic codes, which involve special monomials. The paper by K. Abdukhalikov and S. Mesnager is a nice survey on connections among bent functions that are linear on elements of some classical spreads, semifields, and oval polynomials. In addition, it contains some new results. It should be mentioned that semifields and planar functions are closely linked.

In a nutshell, the papers included in this special issue have enhanced the interplays between special functions and codes, and may stimulate further progress on this topic.

Finally, we thank all the authors for their contributions, Claude Carlet for giving us the opportunity to edit this special issue, Melissa Fearon and others at Springer Verlag for their supports. 